\title{
Ethanol Exposure Alters Protein Expression in a Mouse Model of Fetal Alcohol Spectrum Disorders
}

\author{
Stephen Mason, ${ }^{1}$ Bruce Anthony, ${ }^{1}$ Xianyin Lai, ${ }^{2}$ Heather N. Ringham, ${ }^{2}$ Mu Wang, ${ }^{3,4}$ \\ Frank A. Witzmann, ${ }^{2}$ Jin-Sam You, ${ }^{4}$ and Feng C. Zhou, ${ }^{1,5}$ \\ ${ }^{1}$ Department of Anatomy and Cell Biology, Indiana University School of Medicine, Indianapolis, IN 46202, USA \\ ${ }^{2}$ Department of Cellular and Integrative Physiology, Indiana University School of Medicine, Indianapolis, IN 46202, USA \\ ${ }^{3}$ Department of Biochemistry and Molecular Biology, Indiana University School of Medicine, Indianapolis, IN 46202, USA \\ ${ }^{4}$ Monarch LifeSciences, LLC., Indianapolis, IN 46202, USA \\ ${ }^{5}$ Stark Neuroscience Research Institute, Indiana University School of Medicine, Indianapolis, IN 46202, USA
}

Correspondence should be addressed to Feng C. Zhou, imce100@iupui.edu

Received 17 January 2012; Revised 1 April 2012; Accepted 1 April 2012

Academic Editor: Vladimir Uversky

Copyright ( 2012 Stephen Mason et al. This is an open access article distributed under the Creative Commons Attribution License, which permits unrestricted use, distribution, and reproduction in any medium, provided the original work is properly cited.

\begin{abstract}
Alcohol exposure during development can result in variable growth retardation and facial dysmorphology known as fetal alcohol spectrum disorders. Although the mechanisms underlying the disorder are not fully understood, recent progress has been made that alcohol induces aberrant changes in gene expression and in the epigenome of embryos. To inform the gene and epigenetic changes in alcohol-induced teratology, we used whole-embryo culture to identify the alcohol-signature protein profile of neurulating C6 mice. Alcohol-treated and control cultures were homogenized, isoelectrically focused, and loaded for 2D gel electrophoresis. Stained gels were cross matched with analytical software. We identified 40 differentially expressed protein spots $(P<0.01)$, and 9 spots were selected for LC/MS-MS identification. Misregulated proteins include serotransferrin, triosephosphate isomerase and ubiquitin-conjugating enzyme E2 N. Misregulation of serotransferrin and triosephosphate isomerase was confirmed with immunologic analysis. Alteration of proteins with roles in cellular function, cell cycle, and the ubiquitin-proteasome pathway was induced by alcohol. Several misregulated proteins interact with effectors of the NF- $\kappa$ B and Myc transcription factor cascades. Using a whole-embryo culture, we have identified misregulated proteins known to be involved in nervous system development and function.
\end{abstract}

\section{Introduction}

Fetal alcohol spectrum disorders (FASD) is characterized by a multiorgan phenotype with various degrees of neural developmental deficit, growth retardation, microcephaly, joint deformity, cardiac malformation, and facial dysmorphology due to alcohol exposure during pregnancy $[1,2]$. A continuum of deficits affecting multiple aspects of cognition and behavior is seen in FASD-affected children [3]. While the underlying mechanism driving FASD remains unclear [4-6], altered cellular pathways include cellular metabolism [7-9], suppression of protein and DNA synthesis [10], and oxidative stress [11]. Recent evidence suggests alcohol induces aberrant epigenetic changes during neurogenesis, when proper gene and protein expression plays a critical role in neurodevelopment [12]. For example, we have found aberrant DNA methylation results in the misexpression of neurospecification genes $N g n$ and Sox $5[12,13]$. Over 2,100 differentially methylated genes have been identified in alcohol-treated embryos, suggesting that multiple cellular pathways are likely to be altered at the protein level [13].

Ethanol has multiple effects on methyl donors and inhibits folate-mediated methionine synthesis [14], thereby interrupting the substrate for biologic methylation. A correlation between altered DNA methylation and the severity of neural tube defects is seen in alcohol-treated embryos [13]. Folate supplementation of FASD models can ameliorate alcohol-induced neural tube defects [15] and protein misregulation in the central nervous system (CNS) [16]. These results suggest methionine synthesis, subsequent DNA 
methylation, and the resulting epigenome is critical for normal embryonic development [14]. Interestingly, in gravid mice the methionine synthesis pathway is altered with iron deficiency as well as alcohol exposure [17]. Iron deficiency produces CNS defects, such as microencephaly and psychomotor dysfunction, that parallel the defects seen in FASD [18]. Ethanol also affects the intracellular proteolytic pathway, which is essential for cellular regulation and viability [19]. The ubiquitin-proteasome system is the primary cellular pathway for degrading damaged proteins and controlling the level of gene regulatory factors and has been implicated in the etiology of alcoholic liver disease and neurodegenerative disease, including Alzheimer's and Parkinson's disease [20]. Alcohol-fed rodents accumulate protein in the liver, reflecting a decrease in hepatocyte catabolism [19], ostensibly from downregulation of proteasome-interacting proteins and regulatory complexes [21]. More significantly, microarray and proteomic analyses demonstrate reduced expression of proteasome components in alcohol-exposed embryonic mice [16, 22].

Previous gene expression and proteomic analyses of FASD models have been completed, and the results generally overlap. Microarray analysis of alcohol-exposed fetal brain has demonstrated downregulation of genes involved in cell proliferation, differentiation, apoptosis, and the ubiquitinproteasome pathway [23]. The identified genes have been shown to contribute to tissue growth and remodeling, as well as neuronal growth and survival. Gutala et al. [24] demonstrated that alcohol treatment of mouse cortical cells suppresses the expression of ubiquitin-proteasome and ribosomal pathway genes, leading to disruption of the protein degradation and protein synthesis machinery. Green and colleagues [22] have shown that proteasome and ribosome pathways are downregulated after 3 hours of alcohol exposure at E8; conversely, cellular pathways involved in tight junctions, focal adhesion, and regulation of the actin cytoskeleton are upregulated at $3 \mathrm{hrs}$. Similarly, proteomic analyses have demonstrated alteration of proteins involved in energy production, cellular signaling, protein translation, and the ubiquitin-proteasome system [16]. Specific proteins known to be alcohol-sensitive include brain-derived neurotrophic factor (BDNF) [25], cellular retinoic acid binding protein I (CRABP-I) [26], and proteasome subunit $\alpha$ type6 [16]. Using embryonic cultures, we have previously found upregulation of cyclin D1 and proapoptotic p53 [27].

In the current study, we have used an established wholeembryo culture model with strictly controlled staging (timed by somite number) and alcohol dose to obtain an alcoholsignature protein profile. This profile may inform the gene and epigenetic changes in alcohol-induced teratology and/or lead to the development of biomarkers of prenatal alcohol exposure. Previous research with this culture model has demonstrated alcohol-induced abnormalities of the neural tube, brain vesicles, optic system, heart, and limb buds [13]. Neural tube abnormalities occur most frequently in the head fold and correlate with more severe genetic and phenotypic abnormalities. To characterize misregulated proteins associated with these developmental abnormalities, we have selected 2D gel electrophoresis to investigate the effects of alcohol exposure on the embryonic proteome. The feasibility of whole-embryo 2D gel electrophoresis has been demonstrated in previous studies [28]. Based on our proteomic and immunologic results, we conclude that the epigenetic and morphologic changes induced by alcohol exposure results in protein misregulation during neurulation.

\section{Materials and Methods}

2.1. Embryonic Culture. A whole-embryo culture model was used to study alterations in protein regulation associated with FASD. This FASD model has been described previously by Ogawa et al. [29]. Briefly, C57BL/6 (C6) mice (weighing approximately $20 \mathrm{~g}$ ) were purchased from Harlan, Inc. (Indianapolis, IN, USA). All animal procedures were approved by the Indiana University Institutional Animal Care and Use Committee. Mouse breeders were individually housed and maintained on a 12 hour light-dark cycle (light on: 19:00-7:00) and provided laboratory chow and water ad libitum. When a vaginal plug was detected after the mating period, it was designated as gestational day 0 (GD0) or embryonic day 0 (E0). On E8.25, dams were sacrificed by overdose using $\mathrm{CO}_{2}$ gas. The technique for whole-embryo culture was based on the method described by New [30]. Briefly, the gravid uterus was removed and placed in a sterile phosphate buffer containing saline (PBS, $0.1 \mathrm{M}$ ) at $37^{\circ} \mathrm{C}$. Decidual tissues and the Reichert membrane were removed carefully and immediately immersed in the PBS containing $4 \%$ fetal bovine serum (Sigma, St. Louis, MO, USA), leaving the visceral yolk sac and a small piece of the ectoplacental cone intact. Three embryos bearing three to six somites were placed in a culture bottle $(20 \mathrm{~mL})$ containing the culture medium, which consisted of $70 \%$ immediately centrifuged heat-inactivated rat serum (Harlan Sprague-Dawley, Inc.) and $30 \%$ PB1 buffer $(137 \mathrm{mM} \mathrm{NaCl}, 2.7 \mathrm{mM} \mathrm{KCl}, 0.5 \mathrm{mM}$ $\mathrm{MgCl}_{2}, 8 \mathrm{mM} \mathrm{Na}_{2} \mathrm{HPO}_{4}, 1.47 \mathrm{mM} \mathrm{KH}_{2} \mathrm{PO}_{4}, 0.9 \mathrm{mM} \mathrm{CaCl}$, $5.6 \mathrm{mM}$ glucose, and $0.33 \mathrm{mM}$ sodium pyruvate; $\mathrm{pH}$ 7.4). The embryos were then supplemented with penicillin and streptomycin (20 units $/ \mathrm{mL}$ and $20 \mu \mathrm{g} / \mathrm{mL}$, resp., Sigma). Bottles were gassed at 0 to $22 \mathrm{hr}$ with $5 \% \mathrm{O}_{2}, 5 \% \mathrm{CO}_{2}$, and $90 \% \mathrm{~N}_{2}$ and at 22 to $44 \mathrm{hr}$ with $20 \% \mathrm{O}_{2}, 5 \% \mathrm{CO}_{2}$, and $75 \% \mathrm{~N}_{2}$ in a rotating culture system (B.T.C. Precision Incubator Unit; B.T.C. Engineering, Cambridge, England; $36 \mathrm{rpm})$ at $37^{\circ} \mathrm{C}$. After the preculture period $(2-4 \mathrm{hr}$; maximum, $4 \mathrm{hr}$ ), alcohol exposure was started by transferring the embryos into the medium containing $6 \mu \mathrm{L} / \mathrm{mL}$ of $95 \%$ ethanol (approximately $400 \mathrm{mg} / \mathrm{dL}$ ). Before the experiment, the medium alcohol levels in the setting were tested using an Analox alcohol analyzer (Analox Instruments USA, Lunenburg, MA, USA). The control group was cultured in the medium with no ethanol. On day 2, the culture medium was changed $22 \mathrm{hr}$ after the start of the exposure with the same treatments described above. All cultures were terminated $44 \mathrm{hr}$ from the beginning of treatment. A total of 73 embryos from 25 separate cultures were used in this study. In each culture batch, control embryos were included to avoid misinterpretations produced by any differences in the cultural conditions among these experiments. 
The concentration of ethanol in the medium across $24 \mathrm{hr}$ was tested at 0,12 , and $22 \mathrm{hr}$ (the timing of medium change), previously, in a separate group not used for vulnerability study [29]. The medium alcohol level used in current study is comparable to the in vivo dosage for the lowest teratogenic dose used by Webster et al. [31]. This level is considered within the range attained by human alcoholics [32, 33]. At the end of culture, viability was confirmed by blood circulation of the yolk sac and cardiac activity. Embryos were snap frozen in liquid $\mathrm{N}_{2}$.

2.2. $2 \mathrm{D} \mathrm{Gel} \mathrm{Analysis.} \mathrm{Frozen}\left(-70^{\circ} \mathrm{C}\right)$ embryos were pooled $(n=7)$ in a $25 \mathrm{~mL}$ beaker, along with 8 volumes of a solution containing $9 \mathrm{M}$ urea, 4\% Igepal CA-630 ([octylphenoxy] polyethoxyethanol), 1\% dithiothreitol (DTT), and 2\% carrier ampholytes ( $\mathrm{pH} 8-10.5)$, and thoroughly sonicated. After solubilization at room temperature, samples were centrifuged at $100,000 \times \mathrm{g}$ for 30 min using a Beckman TL100 ultracentrifuge (Fullerton, CA, USA) to remove nucleic acid and insoluble materials; the supernatants were stored at $-45^{\circ} \mathrm{C}$ until $2 \mathrm{DE}$ separation. Using overnight, passive rehydration at room temperature, homogenized sample was loaded onto immobilized pH-gradient (IPG) strips ( $24 \mathrm{~cm}$, nonlinear $\mathrm{pH} 3-10$ : BioRad: Hercules, CA, USA). Isoelectric focusing (IEF) was performed with a Protean IEF Cells (BioRad) (10 strips/instrument), by a program of progressively increasing voltage for a total of 100,000 Vhr. A computer-controlled gradient casting system was used to prepare second-dimension (2D) sodium dodecyl sulfate (SDS) gradient slab gels $(20 \times 25 \times 0.15 \mathrm{~cm})$ in which the acrylamide concentration varied linearly from $11 \%$ to $17 \%$ T. First-dimension IPG strips were loaded directly onto the slab gels following equilibration for $10 \mathrm{~min}$ in buffer I. All nine 2D slab gels were run in parallel at $8^{\circ} \mathrm{C}$ for $18 \mathrm{~h}$ at $160 \mathrm{~V}$ and subsequently fixed and stained using a sensitive colloidal Coomassie Blue G-250 procedure [34]. After $96 \mathrm{~h}$, gels were washed several times with water and scanned at $95.3 \mu \mathrm{m} /$ pixel resolution using a GS-800 Calibrated Imaging Densitometer (Bio-Rad). The resulting 12 bit images were analyzed using PDQuest software (BioRad, v.7.1). Background was subtracted and protein spot density peaks detected and counted. Because total spot counts and the total optical density are directly related to the total protein concentration, individual protein quantities were thus expressed as parts-per-million (ppm) of the total integrated optical density, after normalization against total image density. A reference pattern was constructed and each of the gels in the match set was matched to the reference gel. Numerous proteins that were uniformly expressed in all patterns were used as landmarks to facilitate rapid gel matching. Protein spots that were not present in at least 3 of the gels for both groups were removed from the analyses. A statistical mean was determined for each spot in both the alcohol-treated and control groups. A $t$-test was performed between the two groups to determine which spots had a significantly different staining intensity $(P<0.01)$. Protein spots were excised from the gels and destained with acetonitrile (ACN) in $50 \mathrm{mM} \mathrm{NH}_{4} \mathrm{HCO}_{3}$, reduced with $10 \mathrm{mM}$
DTT, alkylated with iodoacetamide, and in-gel digested with modified trypsin at $37^{\circ} \mathrm{C}$ overnight, followed by serial extraction with ACN. Tryptic peptides were analyzed on a LTQ mass spectrometer (Thermo Electron, San Jose, CA, USA) coupled with an Agilent 1100 HPLC system (Agilent Technologies, Santa Clara, USA) and C18 nanocolumn $(75 \mu \mathrm{m} \times 5 \mathrm{~cm})$. Peptides were eluted with a linear gradient from 5 to $45 \%$ acetonitrile developed over $60 \mathrm{~min}$ at a flow rate of $300 \mathrm{~nL} / \mathrm{min}$. The acquired MS/MS data were filtered and analyzed by a proprietary algorithm [35]. Database searches against the IPI (International Protein Index) mouse database and the nonredundant mouse database were carried out using both the X!Tandem and SEQUEST algorithms.

\subsection{Ingenuity Pathways Analysis. Identified proteins were} submitted to Ingenuity Pathways Analysis (IPA) (Ingenuity, Inc. http://www.ingenuity.com) online software. IPA builds networks based upon information extracted from the scientific literature and deposited in the Ingenuity Knowledge Base. IPA provides an assessment of the signaling and metabolic pathways, molecular networks, and biological processes that are most significantly perturbed in the experimental condition. The Path Designer feature was utilized to abridge the molecular network for publication.

2.4. Western Blot Analysis. Western blotting was used to confirm the misregulation of proteins of mechanistic interest in FASD, as previously described [27]. For total protein procurement, 3 embryos were pooled as one sample. Homogenized embryos were loaded on two, $8-12 \%$ PAGE gels. Proteins were transferred to a nitrocellulose membrane and blocked overnight with $5 \%$ milk and $0.02 \%$ BSA, washed $3 \times$ in TBST, then incubated for $2-4 \mathrm{~h}$ at $30^{\circ} \mathrm{C}$ using the appropriate $1^{\circ}$ antibody. Primary antibodies included: goat poly (rC)-binding protein 1 , rabbit serotransferrin and triosephosphate isomerase (Santa Cruz Biotechnology), and mouse Ube2N (Abcam). Monoclonal anti-GAPDHperoxidase (Sigma) (1:40,000 dilution) was incubated as internal control for densitometry. Membranes are washed and incubated with the appropriate HRP conjugated $2^{\circ}$ antibody and detected with a Pierce ECL detection kit (Thermo Fisher Scientific, Rockford, IL, USA). Blots are detected with a FOTO/Analyst Luminary/FX system (FOTODYNE Inc., Hartland, WI, USA), and densitometric comparisons were made with TotalLab Quant software (v.11.5). GAPDH density measurements were used for load controls, and variations in measurements were adjusted by percentage to the highest values for that membrane. Measurements for each target protein were then adjusted in accordance with the GAPDH load controls. All changes in protein expression from alcohol treatment are reported as a percentage change compared to controls, with a minimum of 3 samples/treatment group. Statistical analysis used Student's $t$-test on Microsoft Excel (v. 14).

2.5. Immunohistochemistry Analysis. The embryos of the two treatment groups (alcohol $=4$, control $=4$ embryos) were paired and embedded in gelatin together at E10.25, 
with careful alignment of their level and orientation. They were sectioned in 40 micron and processed for immunohistochemistry in parallel; this practice avoids any bias through the staining procedure and is convenient for comparing levels of embryo sections side by side. All sections were then washed $3 \times$ in PBS. They were then incubated in (1) $3 \% \mathrm{H}_{2} \mathrm{O}_{2}(\mathrm{v} / \mathrm{v})$ in PBS for 30 minutes and (2) PBS containing 4\% normal control serum, and $0.3 \%$ Triton X-100 (TX) for 30 minutes at room temperature to block nonspecific binding. Sections were next incubated with antibodies against serotransferrin $(1: 50)$ and triosephosphate isomerase (1:50). All $1^{\circ}$ antibodies were incubated overnight at room temperature. The sections were then incubated with a $2^{\circ}$ antibody $(1: 250$, Jackson ImmunoResearch Laboratory, West Grove, PA) for $1 \mathrm{hr}$, followed by an avidin-biotin peroxidase complex (Vector Laboratories) for $1 \mathrm{hr}$ at room temperature. All sections were washed $3 \times$ with PBS for 5 minutes between each antibody incubation. The color reaction (brown) was developed by adding $0.05 \% 3^{\prime}$ to $3^{\prime}$-diaminobenzidine tetrahydrochloride and $0.003 \% \mathrm{H}_{2} \mathrm{O}_{2}$ in Tris buffer to reveal the peroxidase activity.

\section{Results}

3.1. Embryonic Growth Retardation/Abnormalities. Among all cultured embryos, more than $95 \%$ maintained active heartbeats and blood circulation during the incubation period, and only those were used for analysis. Control and alcohol-treated embryos are shown in Figure 1. Embryonic development was significantly compromised by alcoholexposure, consistent with previous studies [12, 13, 36, 37]. Growth retardation and abnormalities of the brain, heart, and limbs were seen in alcohol-treated embryos. Specific abnormalities include neural tube defects, heart malformations, small/slanted eyes, abnormal tail morphology, and unfinished turning of the neural axis. A reduced blood/ vascular system with less yolk sac vascularity and a lower red coloration was apparent in the alcohol-treated versus control embryos.

3.2. $2 \mathrm{D} \mathrm{Gel} \mathrm{Analysis.} \mathrm{The} \mathrm{total} \mathrm{number} \mathrm{of} \mathrm{gel} \mathrm{spots} \mathrm{detected}$ by PDQuest was 2,050. Forty gel spots with significant $(P<$ 0.01 ) differential expression ( $>1.5$-fold change) between alcohol-treated and control embryos were identified. Integrated densities of 8 gel spots fell below $200 \mathrm{ppm}$, a protein density that is too low for reliable MS/MS identification. Of the remaining 32 spots, 24 were downregulated and 8 were upregulated in alcohol-treated embryos. To prioritize the study, only those spots with a highly significant $(P<$ 0.007 ) difference in expression levels were selected for analysis. Five downregulated and 4 upregulated spots were selected for LC-MS/MS identification (Figure 2). Table 1 lists the numerical assignment, number of peptides used for identification, UniProtKB accession number, gene name, and the protein identification for each spot with a significant $(P<0.007)$ difference in expression levels between the two groups.

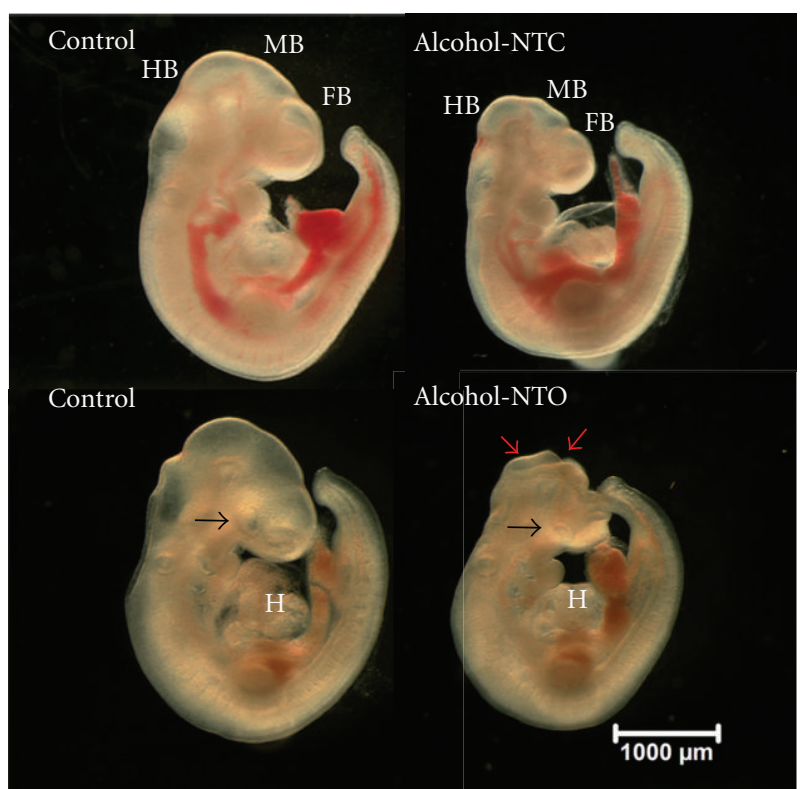

FIGURE 1: Alcohol-induced dysmorphology of embryonic development at E10.25. The alcohol-treated embryos (right side of figure) showed developmental delay and abnormalities. Examples are demonstrated in small forebrain (FB), midbrain $(\mathrm{MB})$, or hindbrain (HB); abnormality of the heart $(\mathrm{H})$; small eyes (black arrows) as compared with control (left). A reduced blood/vascular system with lower red coloration was apparent in the alcoholtreated embryos. A major dichotomic phenotype is embryos with neural tube closed but with delay and abnormality in development (Alcohol-NTC), and neural tube opened (Alcohol-NTO). An open neural tube is shown (red arrows) in an alcohol-treated embryo (bottom right). Scale bar: 1,000 micron.

3.3. Western Blot Analysis. Western blot image density analysis confirmed upregulation of serotransferrin (Tf) and triosephosphate isomerase (Tpi1) $(P<0.05)$ (Figure 3). Poly (rC)-binding protein 1 (Pcbp1) and ubiquitin-conjugating enzyme E2 N (Ube2N) are downregulated on 2D gel analysis, but this was not statistically significant by Western blot densitometry. The Ube2n band is faint.

3.4. Immunohistochemistry. Immunostaining (im) of E10.25 embryos demonstrated upregulation of $\mathrm{Tf}$ and Tpil in alcohol-treated embryos. Tf-im consistently localized to the heart, branchial arches and somites of treated embryos relative to control (Figure 4). Tpi1-im was diffusely increased; however, this staining pattern was less consistent among treated embryos.

3.5. Ingenuity Pathways Analysis. Nine proteins identified by LC-MS/MS were submitted to IPA to assess the biological networks perturbed by alcohol treatment (Figure 5). The top reported networks were cell cycle, cell death, gene expression and reproductive system development and function. The top reported biological functions were antigen presentation, cell cycle, cellular signaling, cellular function and maintenance, hematopoiesis, and immune cell trafficking. The 
TAble 1: Protein identification of differentially expressed spots from 2D gel analysis of alcohol-treated embryos. The spot assignments, number of peptides used for identification, UniProtKB accession number, gene name, and the protein identification for each spot. Proteins are grouped by their pattern of expression (up- or downregulated) in alcohol-treated embryos relative to control.

\begin{tabular}{|c|c|c|c|c|}
\hline Spot ID & No. peptides & Accession & Gene ID & Protein ID \\
\hline \multicolumn{5}{|c|}{ Proteins upregulated in alcohol-treated embryos } \\
\hline 5829 & 54 & Q921I1 & $T f$ & Serotransferrin precursor \\
\hline 8217 & 9 & Q9WVA4 & Tagln 2 & Transgelin-2 \\
\hline 4601 & 8 & Q9QZ83 & Actg1 & Gamma actin-like protein \\
\hline 7324 & 5 & P17751 & Tpil & Triosephosphate isomerase \\
\hline \multicolumn{5}{|c|}{ Proteins downregulated in alcohol-treated embryos } \\
\hline 7605 & 54 & P60335 & Pcbp1 & Poly $(\mathrm{rC})$-binding protein 1 \\
\hline 4424 & 36 & P70195 & Psmb7 & $\begin{array}{c}\text { Proteasome subunit } \beta \\
\text { type-7 }\end{array}$ \\
\hline 4110 & 21 & P61089 & Ube $2 n$ & $\begin{array}{l}\text { Ubiquitin-conjugating } \\
\text { enzyme E2 N }\end{array}$ \\
\hline 7013 & 16 & Q9CQW5 & Lgals2 & Galectin-2 \\
\hline 5112 & 7 & Q99L47 & St13 & Hsc70-interacting protein \\
\hline
\end{tabular}

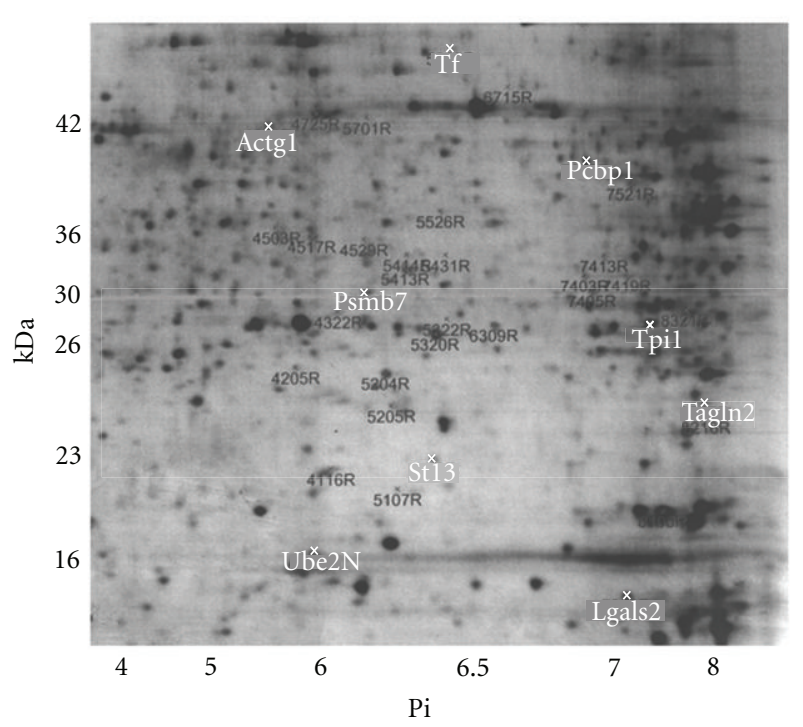

Figure 2: Sample 2D gel separation of whole-embryo proteins. Whole-embryos were solubilized and the proteins separated using IPG strips in the 1st dimension and SDS gradient slab gels in the 2nd dimension. The protein profiles were visualized by Coomassie blue staining. Nine MS/MS identified protein spots with differential expression due to alcohol treatment are highlighted with a white " $\mathrm{x}$ " and labeled with the gene name. The remaining spots with altered expression are labeled with the spot number in black font followed by "R," signifying this gel as the reference (R) control.

top canonical pathways affected by alcohol treatment are clathrin-mediated endocytosis signaling and the ubiquitinproteasome pathway.

\section{Discussion}

This study was designed to characterize the alcohol-signature protein profile of a whole-embryo culture model of FASD.
The whole-embryo was studied at an early, neurulating stage, and the resulting protein profile represents an average across all cell types and tissues. While the embryo system is less sensitive for identifying misregulated proteins in individual organs, our results clearly demonstrate that this model is sensitive for detecting systemic protein misregulation. The analysis detected 40 differentially expressed protein spots, and 9 spots were identified by MS/MS. The possibility exists that some of these protein expression changes may be posttranslational modifications (PTM); nevertheless, these PTM might be important to the pathology of FASD and could be characterized in subsequent studies.

The abnormal embryonic development resulting from the alcohol treatment is consistent with our previous report [29]. The affected structures were derived from each of the three germ layers and involve a wide range of tissues and organs. These effects on protein regulation may parallel the growth delay and developmental abnormalities including brain, neural tube, eye, heart, blood cells, and embryonic vascularization, which are major targets in FASD [13].

As explained in the methods section, 9 differentially expressed proteins were submitted for Ingenuity analysis. Perturbations in cellular function and maintenance, cell cycle, and the ubiquitin-proteasome pathway were demonstrated by Ingenuity. Biological functions altered by alcohol include hematopoiesis and clathrin-mediated endocytosis. We have previously confirmed absent expression of 4 key hematopoietic genes ( $A d d 2, B 2 m, C p$, and $G p a)$, along with an embryonic phenotype consistent with reduced hematopoiesis in alcohol-exposed embryos [12]. Previous studies have demonstrated perturbations of clathrinmediated endocytosis in adult rat brain extracts [38]; this may result in alterations of postsynaptic plasticity. The Ingenuity molecular network (Figure 5) suggests that the NF- $\kappa$ B cascade and transcription factor Myc may be specifically involved in the pathogenesis of FASD. Five ethanol sensitive proteins interact with Protein IKBKE, a kinase that phosphorylates inhibitors of NF- $\kappa \mathrm{B}$ and leads 


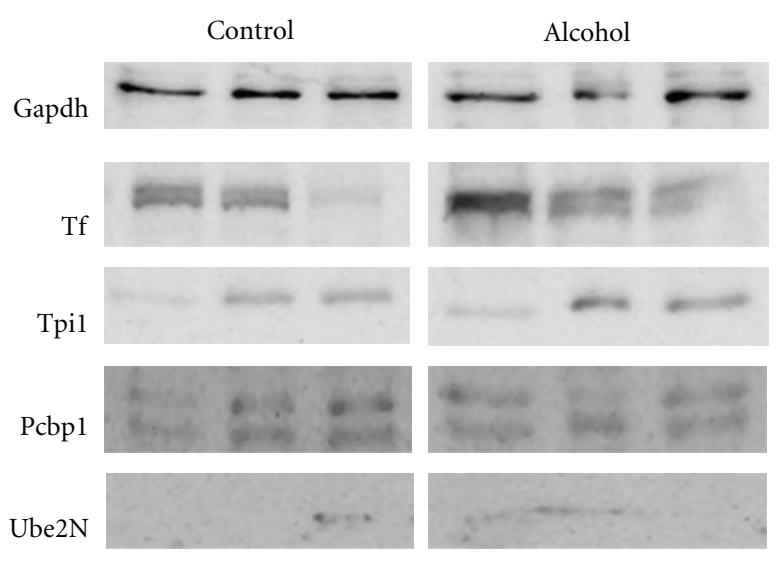

(a)

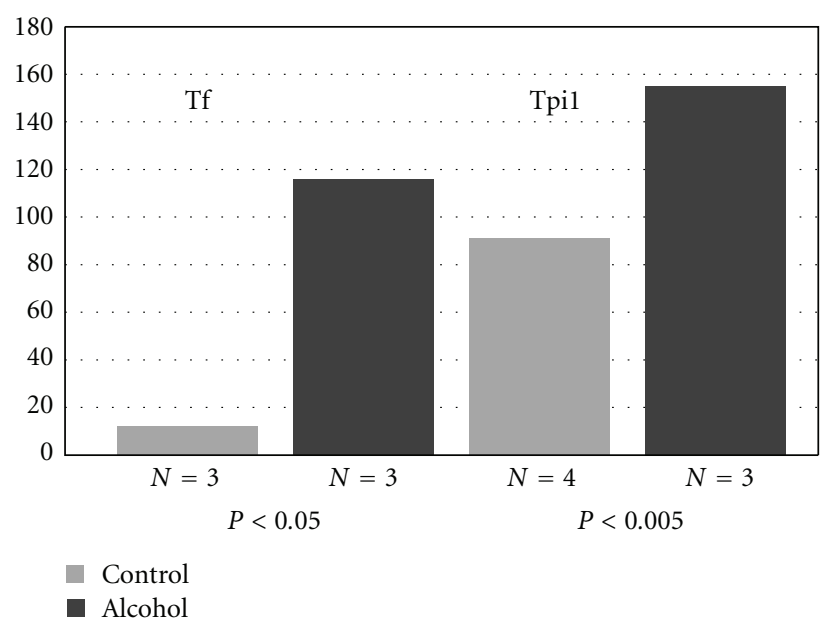

(b)

Figure 3: Misregulated proteins of mechanistic interest in FASD were confirmed with Western blotting. Both alcohol and control protein $(20 \mu \mathrm{g})$ samples were run on SDS-PAGE gels and detected by chemical luminescence. GAPDH was used as an internal control. Statistical analysis of band density by Student's $t$-test showed that alcohol upregulates serotransferrin (Tf) and triosephosphate isomerase (Tpil) $(P \leq 0.05)$.

to NF- $\kappa \mathrm{B}$ activation [39]. The transcription factor NF$\kappa \mathrm{B}$ protects cells against DNA damage-induced cell death and may play a role in the immune response. Alcohol differentially regulates NF- $\kappa$ B activity in various tissues [40]. Ethanol activates $\mathrm{NF}-\kappa \mathrm{B}$ in cerebral vascular muscle cells [41], in contrast, ethanol diminishes NF- $\kappa \mathrm{B}$ target gene expression in fetal rhombencephalic neurons via a 5-HT(1A) (serotonin) mediated pathway [42]. Ethanol also interferes with inflammatory cytokine production by downregulating $\mathrm{NF}-\kappa \mathrm{B}$ in monocytes, resulting in impaired immunity $[42,43]$. The transcription factor Myc activates growthrelated genes [39]. Figure 5 shows that Pcbp1 and Tagln2 directly bind with Myc. Ethanol can induce release of intracellular calcium that stimulates the cell cycle and the expression of Myc proteins associated with cell proliferation. Increased proliferation is advantageous during reimplantation, but ethanol interference with terminal differentiation during organogenesis may underlie alcohol teratogenicity [44].

Serotransferrin (Tf) is upregulated by proteomic and immunologic analysis (Figures 2-4), which may be a compensatory response to the altered iron regulation seen with alcohol exposure. Tf transports iron from tissue sites of absorption to sites of storage or utilization and has a role in stimulating cell proliferation [39]. Because iron is an essential cofactor in neurotransmitter synthesis and myelination, maternal iron status is an important modulator of fetal alcohol-induced neurobehavioral damage $[45,46]$. Interestingly, fetal alcohol syndrome produces defects that parallel the abnormalities of early iron deficiency, including microcephaly and motor and behavioral deficits [18]. Our observation that $\mathrm{Tf}$ localizes to the heart, branchial arches and somites with alcohol treatment is interesting (Figure 4).
These findings warrant further investigation into the association between altered iron regulation, cardiac malformation, and FASD.

Protein triosephosphate isomerase (Tpil) and transgelin $($ Tagln2) were upregulated in our proteomic analysis. Tpi1 misregulation was confirmed with Western blot. Diffuse Tpil-im was seen on whole-embryo immunohistochemistry (Figure 4). Our previous proteomic analysis demonstrated upregulation of Tpil in the nucleus accumbens of alcoholexposed adult rats [47]. Green et al. noted upregulation of pentose phosphate pathway genes in alcohol-exposed C6 mouse embryos [22]. Tpil is an enzyme of the pentose phosphate pathway that binds to microtubules and other subcellular components. Protein Tpil can reduce the enzymatic activity of other metabolic proteins when bound to them [48]. Tagln 2 is one of the earliest markers of differentiated smooth muscle. The function of Tagln 2 has not yet been determined, but proteomic analysis has demonstrated altered regulation during neurulation $[49,50]$.

The protein ubiquitination pathway targets molecules for proteasomal degradation through conjugation with Lys48-linked ubiquitin. Alternatively, proteins modified with Lys63-linked ubiquitin chains play roles in signal transduction through the NF- $\kappa \mathrm{B}$ cascade, receptor endocytosis, and DNA-repair processes [51]. Degradation of damaged proteins is critical for cellular viability, and evidence suggests ethanol can downregulate components of the ubiquitinproteasome system [19]. Previous studies have shown that proteasome $\beta$ type- 7 is downregulated at the gene and protein level in a murine model of FASD $[16,22]$. Our 2 D gel analysis also demonstrates downregulation of proteasome $\beta$ type-7. This proteasome subunit forms the catalytic core of the 26S proteasome [19], the main mechanistic component of intracellular proteolysis. 


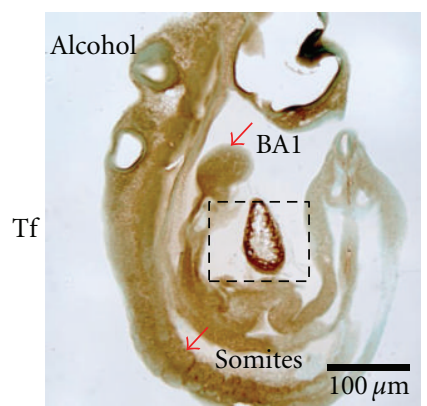

(a)

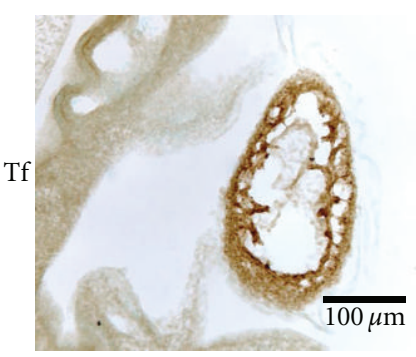

(c)

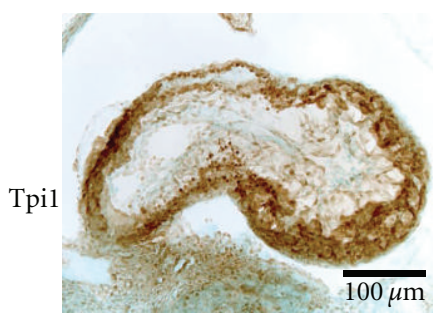

(e)

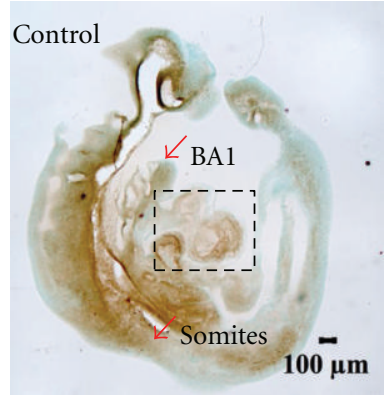

(b)

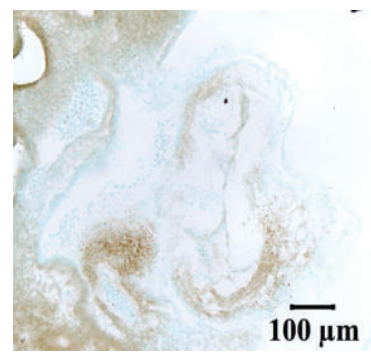

(d)

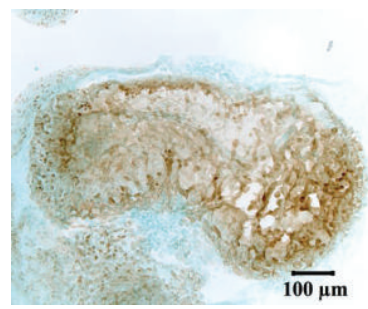

(f)
FIGURE 4: Immunolocalization of serotransferrin to the heart, branchial arches and somites with alcohol treatment. Serotransferrin immunostaining (Tf-im) (a,b,c,d) and triosephosphate isomerase immunostaining (Tpil-im) (e,f) are increased in alcoholtreated embryos at E10.25. Tf-im consistently localized to the heart (dotted region), branchial arch 1 (BA1) and somites (somites) of alcohol-treated embryos (a) relative to control (b). High magnification of the dotted region is shown in (c) (Alcohol) and (d) (Control). Tpil-im was diffusely increased in alcohol-treated embryos (e) relative to control (f), but the staining pattern was less consistent.

Our 2D gel analysis demonstrated downregulation of ubiquitin-conjugating enzyme E2 N (Ube2N) with alcoholexposure, congruent with our previous analysis of adult rat brain extracts [47]. A gene expression analysis demonstrated that $U b e 2 b$, a member of the ubiquitin-conjugating enzyme family, was downregulated in neurulating embryos [12]. Ube $2 \mathrm{~N}$ and Ube2b both catalyze the synthesis of Lys63-linked poly-ubiquitin chains and are involved in the postreplication repair of damaged DNA. Interestingly, a Drosophila homolog of Ube $2 \mathrm{~N}$ was originally isolated as bendless protein, whose mutation affects neuronal synaptic connectivity and visual system development [52-54]. As shown in Figure 5, Ube2N binds with inhibitor of nuclear factor kappa-B kinase subunit $\varepsilon$ (Protein IKBKE), a kinase

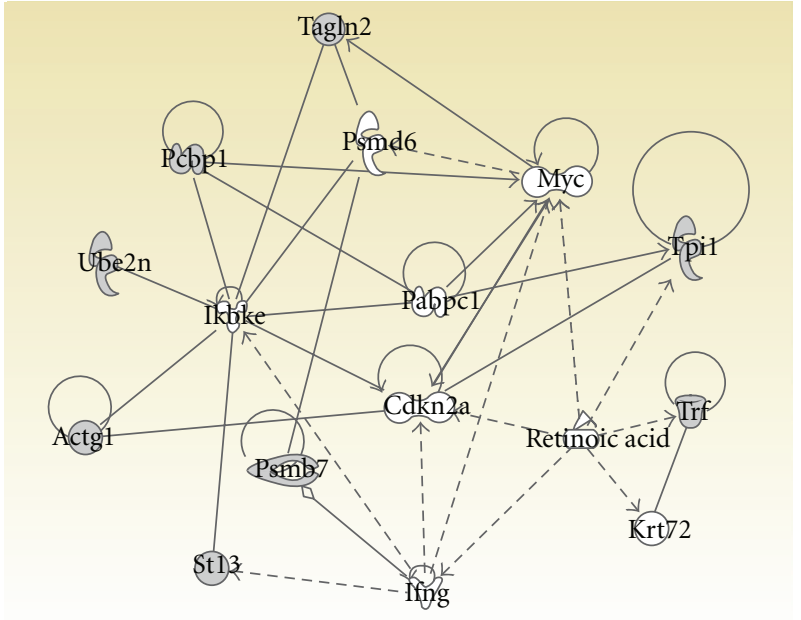

(c) 20002010 ingenuity system, inc. all rights reserved.

Figure 5: Ingenuity Pathways Analysis of misregulated proteins from alcohol-treated whole-embryo culture. Proteins with altered regulation were analyzed with Ingenuity Pathways Analysis online software. This Core Analysis highlights the signaling and metabolic pathways, molecular networks, and biological processes that are most significantly perturbed in the alcoholtreated cultures. The Ingenuity Path Designer feature was utilized to abridge the molecular network. The proteins are represented by shapes that reflect protein function (https://analysis.ingenuity.com/pa/info/help/help.htm\#legend.htm) and labeled with their gene name. Proteins identified in alcohol-treated embryos have shaded shapes; molecules not identified in this study have clear shapes. These molecules were included in the abridged network if they were linked to multiple identified proteins or formed integral links between identified proteins. Solid lines indicate direct interactions and dashed lines are indirect interactions. Abbreviations: Actg1-Gamma actin; Cdkn2a-Cyclin-dependent kinase inhibitor 2A; Ifng - Interferon gamma; Ikbke-Inhibitor of kappa b kinase epsilon; Krt72-Keratin 72; Myc — $\mathrm{v}$-myc myelocytomatosis viral oncogene homolog (avian); Pabpcl—poly(A) binding protein, cytoplasmic 1; Pcbp1-poly(rC) binding protein 1; Psmb7Proteasome subunit $\beta$ type 7; Psmd6-Proteasome 26S subunit, non-ATPase, 6; St13- Hsp70 interacting protein; Tagln2Transgelin 2; Trf (Tf)—Serotransferrin; Tpil-Triosephosphate isomerase 1; Ube2n-Ubiquitin-conjugating enzyme E2N.

that activates the NF- $\kappa \mathrm{B}$ pathway. The Western blot analysis demonstrated a faint band at the expected molecular weight of Ube2N $(\sim 17 \mathrm{kDa})$ (Figure 3$)$. Ube $2 \mathrm{~N}$ gene expression is temporally regulated during development [55], and our results suggest that Ube $2 \mathrm{~N}$ protein is only marginally expressed at E10.

Hypoxia and ischemia regulate the expression of several important genes at the level of transcription and of mRNA stability. An isoform of poly (rC)-binding protein (Protein Pcbp1), previously identified as an RNA-binding protein, binds to a hypoxia-inducible protein-binding site in the $3^{\prime}$ region of erythropoietin mRNA and regulates stability $[56,57]$. Protein Pcbp1 is downregulated in our proteomic analysis; however, Western blot did not demonstrate significant misregulation. The embryonic phenotype is consistent with reduced hematopoiesis in this FASD model [12]. 
Since Pcbp1 protects erythropoietin mRNA, we hypothesize that downregulation of Pcbp1 may be the mechanism for decreased hematopoiesis.

Galectin-2, which is expressed in differentiated enteric tissue [58], is downregulated in our analysis. Previous experiments in Xenopus embryos have shown that alcohol exposure causes fetal enteric damage and reduced body length [59]. We hypothesize that downregulation of galectin2 in our FASD model reflects gut underdevelopment.

In summary, alcohol exposure during the period of early neurulation results in an alcohol-signature protein profile. This profile should contribute to (1) generation of testable new hypotheses concerning the mechanistic pathway from protein misregulation to alcohol-induced teratogenesis and (2) development of clinical biomarkers of prenatal alcohol exposure. We identified proteins (serotransferrin, Ube2N) known to be involved in development of the nervous system, and that the NF- $\kappa$ B cascade and the transcription factor Myc may be specifically involved in alcohol-induced teratology. These misregulated proteins may reflect altered cellular pathways secondary to epigenetic, posttranscriptional, or morphologic changes.

\section{Abbreviations}

$\begin{array}{ll}\text { 2D: } & \text { Two-dimensional } \\ \text { ACN: } & \text { Acetonitrile } \\ \text { BDNF: } & \text { Brain-derived neurotrophic factor } \\ \text { BSA: } & \text { Bovine serum albumin } \\ \text { CNS: } & \text { Central nervous system } \\ \text { CRABP-I: } & \text { Cellular retinoic acid binding protein I } \\ \text { DTT: } & \text { Dithiothreitol } \\ \text { FASD: } & \text { Fetal alcohol spectrum disorder } \\ \text { HRP: } & \text { Horse radish peroxidase } \\ \text { Im: } & \text { Immunostaining } \\ \text { IPA: } & \text { Ingenuity Pathways Analysis } \\ \text { IPG: } & \text { Immobilized pH-gradient } \\ \text { IPI: } & \text { International Protein Index } \\ \text { IEF: } & \text { Isoelectric focusing } \\ \text { LC-MS/MS: } & \text { Liquid chromatography-tandem mass } \\ & \text { spectrometry } \\ \text { PTM: } & \text { Post-translational modification } \\ \text { TBST: } & \text { Tris-buffered saline with Tween. }\end{array}$

\section{Acknowledgments}

This paper was supported by NIH P50AA07611 (FAS component) to FCZ. The authors thank Lijun Ni for assistance with embryo culture and immunohistochemistry and Yuanyuan Chen for photography and image labels. The authors thank the Teresita Bellido Laboratory and Brian Dunphy (FOTODYNE, Inc.) for technical assistance with Western blots. The authors have no relevant financial affiliations to disclose.

\section{References}

[1] K. L. Moore and T. V. N. Persaud, The Developing Human: Clinically Oriented Embryology, Saunders, Philadelphia, Pa, USA, 5th edition, 1993.
[2] I. Autti-Rämö, Å. Fagerlund, N. Ervalahti, L. Loimu, M. Korkman, and H. E. Hoyme, "Fetal alcohol spectrum disorders in Finland: clinical delineation of 77 older children and adolescents," American Journal of Medical Genetics, vol. 140, no. 2, pp. 137-143, 2006.

[3] C. Guerri, A. Bazinet, and E. P. Riley, "Foetal alcohol spectrum disorders and alterations in brain and behaviour," Alcohol and Alcoholism, vol. 44, no. 2, pp. 108-114, 2009.

[4] C. R. Goodlett and K. H. Horn, "Mechanisms of alcoholinduced damage to the developing nervous system," Alcohol Research and Health, vol. 25, no. 3, pp. 175-184, 2001.

[5] C. R. Goodlett, K. H. Horn, and F. C. Zhou, "Alcohol teratogenesis: mechanisms of damage and strategies for intervention," Experimental Biology and Medicine, vol. 230, no. 6, pp. 394406, 2005.

[6] E. P. Riley, J. D. Thomas, C. R. Goodlett et al., "Fetal alcohol effects: mechanisms and treatment," Alcoholism, vol. 25, no. 5, supplement, pp. 110S-116S, 2001.

[7] P. Fattoretti, C. Bertoni-Freddari, T. Casoli, G. Di Stefano, G. Giorgetti, and M. Solazzi, "Ethanol-induced decrease of the expression of glucose transport protein (Glut3) in the central nervous system as a predisposing condition to apoptosis: the effect of age," Annals of the New York Academy of Sciences, vol. 1010, pp. 500-503, 2003.

[8] A. K. Snyder, F. Jiang, and S. P. Singh, "Effects of ethanol on glucose utilization by cultured mammalian embryos," Alcoholism, vol. 16, no. 3, pp. 466-470, 1992.

[9] M. W. Miller and D. L. Dow-Edwards, "Structural and metabolic alterations in rat cerebral cortex induced by prenatal exposure to ethanol," Brain Research, vol. 474, no. 2, pp. 316326, 1988.

[10] I. A. Shibley Jr. and S. N. Pennington, "Metabolic and mitotic changes associated with the fetal alcohol syndrome," Alcohol and Alcoholism, vol. 32, no. 4, pp. 423-434, 1997.

[11] C. Guerri, C. Montoliu, and J. Renau-Piqueras, "Involvement of free radical mechanism in the toxic effects of alcohol: implications for fetal alcohol syndrome," Advances in Experimental Medicine and Biology, vol. 366, pp. 291-305, 1994.

[12] F. C. Zhou, Q. Zhao, Y. Liu et al., "Alteration of gene expression by alcohol exposure at early neurulation," BMC Genomics, vol. 12, article 124, 2011.

[13] Y. Liu, Y. Balaraman, G. Wang, K. P. Nephew, and F. C. Zhou, "Alcohol exposure alters DNA methylation profiles in mouse embryos at early neurulation," Epigenetics, vol. 4, no. 7, pp. 500-511, 2009.

[14] J. B. Mason and S. W. Choi, "Effects of alcohol on folate metabolism: implications for carcinogenesis," Alcohol, vol. 35, no. 3, pp. 235-241, 2005.

[15] L. L. Wang, Z. Zhang, Q. Li et al., "Ethanol exposure induces differential microRNA and target gene expression and teratogenic effects which can be suppressed by folic acid supplementation," Human Reproduction, vol. 24, no. 3, pp. 562-579, 2009.

[16] Y. Xu, Y. Tang, and Y. Li, "Effect of folic acid on prenatal alcohol-induced modification of brain proteome in mice," British Journal of Nutrition, vol. 99, no. 3, pp. 455-461, 2008.

[17] N. El Banna, M. F. Picciano, and J. Simon, "Effects of chronic alcohol consumption and iron deficiency on maternal folate status and reproductive outcome in mice," Journal of Nutrition, vol. 113, no. 10, pp. 2059-2070, 1983.

[18] M. W. Miller, A. J. I. Roskams, and J. R. Connor, "Iron regulation in the developing rat brain: effect of in utero 
ethanol exposure," Journal of Neurochemistry, vol. 65, no. 1, pp. 373-380, 1995.

[19] T. M. Donohue Jr., "The ubiquitin-proteasome system and its role in ethanol-induced disorders," Addiction Biology, vol. 7, no. 1, pp. 15-28, 2002.

[20] J. Lowe, A. Blanchard, K. Morrell et al., "Ubiquitin is a common factor in intermediate filament inclusion bodies of diverse type in man, including those of Parkinson's disease, Pick's disease, and Alzheimer's disease, as well as Rosenthal fibres in cerebellar astrocytomas, cytoplasmic bodies in muscle, and Mallory bodies in alcoholic liver disease," Journal of Pathology, vol. 155, no. 1, pp. 9-15, 1988.

[21] M. P. Bousquet-Dubouch, S. Nguen, D. Bouyssié et al., "Chronic ethanol feeding affects proteasome-interacting proteins," Proteomics, vol. 9, no. 13, pp. 3609-3622, 2009.

[22] M. L. Green, A. V. Singh, Y. Zhang, K. A. Nemeth, K. K. Sulik, and T. B. Knudsen, "Reprogramming of genetic networks during initiation of the Fetal Alcohol Syndrome," Developmental Dynamics, vol. 236, no. 2, pp. 613-631, 2007.

[23] M. L. Hard, M. Abdolell, B. H. Robinson, and G. Koren, "Gene-expression analysis after alcohol exposure in the developing mouse," Journal of Laboratory and Clinical Medicine, vol. 145, no. 1, pp. 47-54, 2005.

[24] R. Gutala, J. Wang, S. Kadapakkam, Y. Hwang, M. Ticku, and M. D. Li, "Microarray analysis of ethanol-treated cortical neurons reveals disruption of genes related to the ubiquitinproteasome pathway and protein synthesis," Alcoholism, vol. 28, no. 12, pp. 1779-1788, 2004.

[25] M. Incerti, J. Vink, R. Roberson, I. Benassou, D. Abebe, and C. Y. Spong, "Prevention of the alcohol-induced changes in brain-derived neurotrophic factor expression using neuroprotective peptides in a model of fetal alcohol syndrome," American Journal of Obstetrics and Gynecology, vol. 202, no. 5, pp. 457.e1-457.e4, 2010.

[26] F. C. Zhou and L. N. Wei, "Expression of cellular retinoc acidbinding protein I is specific to neurons in adult transgenic mouse brain," Gene Expression Patterns, vol. 1, no. 1, pp. 6772, 2001.

[27] B. Anthony, F. C. Zhou, T. Ogawa, C. R. Goodlett, and J. Ruiz, "Alcohol exposure alters cell cycle and apoptotic events during early neurulation," Alcohol and Alcoholism, vol. 43, no. 3, pp. 261-273, 2008.

[28] N. D. E. Greene, K. Y. Leung, R. Wait, S. Begum, M. J. Dunn, and A. J. Copp, "Differential protein expression at the stage of neural tube closure in the mouse embryo," The Journal of Biological Chemistry, vol. 277, no. 44, pp. 41645-41651, 2002.

[29] T. Ogawa, M. Kuwagata, J. Ruiz, and F. C. Zhou, “Differential teratogenic effect of alcohol on embryonic development between C57BL/6 and DBA/2 mice: a new view," Alcoholism, vol. 29, no. 5, pp. 855-863, 2005.

[30] D. A. New, "Whole-embryo culture and the study of mammalian embryos during organogenesis," Biological reviews of the Cambridge Philosophical Society, vol. 53, no. 1, pp. 81-122, 1978.

[31] W. S. Webster, D. A. Walsh, S. E. McEwen, and A. H. Lipson, "Some teratogenic properties of ethanol and acetaldehyde in C57BL/6J mice: implications for the study of the fetal alcohol syndrome," Teratology, vol. 27, no. 2, pp. 231-243, 1983.

[32] F. Hoffman, "Generalized depressants of the central nervous system," in A Handbook of Drug and Alcohol Abuse, F. Hoffman and A. Hoffman, Eds., pp. 95-128, Oxford University Press, New York, NY, USA, 1975.
[33] B. Lindblad and R. Olsson, "Unusually high levels of blood alcohol?" JAMA, vol. 236, no. 14, pp. 1600-1602, 1976.

[34] G. Candiano, M. Bruschi, L. Musante et al., "Blue silver: a very sensitive colloidal Coomassie G-250 staining for proteome analysis," Electrophoresis, vol. 25, no. 9, pp. 1327-1333, 2004.

[35] R. E. Higgs, M. D. Knierman, V. Gelfanova, J. P. Butler, and J. E. Hale, "Comprehensive label-free method for the relative quantification of proteins from biological samples," Journal of Proteome Research, vol. 4, no. 4, pp. 1442-1450, 2005.

[36] B. Anthony, S. Vinci-Booher, L. Wetherill, R. Ward, C. Goodlett, and F. C. Zhou, "Alcohol-induced facial dysmorphology in C57BL/6 mouse models of fetal alcohol spectrum disorder," Alcohol, vol. 44, no. 7-8, pp. 659-671, 2010.

[37] Y. Chen, N. C. Ozturk, L. Ni, C. Goodlett, and F. C. Zhou, "Strain differences in developmental vulnerability to Alcohol exposure via embryo culture in mice," Alcoholism, vol. 35, no. 7, pp. 1293-1304, 2011.

[38] W. J. McBride, J. A. Schultz, M. W. Kimpel et al., "Differential effects of ethanol in the nucleus accumbens shell of alcoholpreferring (P), alcohol-non-preferring (NP) and Wistar rats: a proteomics study," Pharmacology Biochemistry and Behavior, vol. 92, no. 2, pp. 304-313, 2009.

[39] "Reorganizing the protein space at the Universal Protein Resource (UniProt)," Nucleic Acids Research, vol. 40, pp. D71D75, 2012.

[40] Y. Peng, K. H. H. Kwok, P. H. Yang et al., "Ascorbic acid inhibits ROS production, NF- $\kappa \mathrm{B}$ activation and prevents ethanol-induced growth retardation and microencephaly," Neuropharmacology, vol. 48, no. 3, pp. 426-434, 2005.

[41] B. M. Altura, A. Gebrewold, A. Zhang, and B. T. Altura, "Ethanol induces rapid lipid peroxidation and activation of nuclear factor-kappa B in cerebral vascular smooth muscle: relation to alcohol-induced brain injury in rats," Neuroscience Letters, vol. 325, no. 2, pp. 95-98, 2002.

[42] M. J. Druse, N. F. Tajuddin, R. A. Gillespie, and P. Le, "The effects of ethanol and the serotonin1A agonist ipsapirone on the expression of the serotonin1A receptor and several antiapoptotic proteins in fetal rhombencephalic neurons," Brain Research, vol. 1092, no. 1, pp. 79-86, 2006.

[43] P. Mandrekar, D. Catalano, and G. Szabo, "Alcohol-induced regulation of nuclear regulatory factor- $\kappa \beta$ in human monocytes," Alcoholism, vol. 21, no. 6, pp. 988-994, 1997.

[44] D. R. Armant and D. E. Saunders, "Exposure of embryonic cells to alcohol: contrasting effects during preimplantation and postimplantation development," Seminars in Perinatology, vol. 20, no. 2, pp. 127-139, 1996.

[45] J. R. Connor, "Iron acquisition and expression of iron regulatory proteins in the developing brain: manipulation by ethanol exposure, iron deprivation and cellular dysfunction," Developmental Neuroscience, vol. 16, no. 5-6, pp. 233-247, 1994.

[46] T. D. Tran et al., "Maternal iron deficiency produces differential learning outcomes and cell loss in a rat model of fetal alcohol spectrum disorders," Alcoholism, vol. 35, article 38A, 2011.

[47] R. L. Bell, M. W. Kimpel, Z. A. Rodd et al., "Protein expression changes in the nucleus accumbens and amygdala of inbred alcohol-preferring rats given either continuous or scheduled access to ethanol," Alcohol, vol. 40, no. 1, pp. 3-17, 2006.

[48] F. Orosz, G. Wágner, K. Liliom et al., "Enhanced association of mutant triosephosphate isomerase to red cell membranes and to brain microtubules," Proceedings of the National Academy of Sciences of the United States of America, vol. 97, no. 3, pp. 1026-1031, 2000. 
[49] R. Hoffrogge, S. Mikkat, C. Scharf et al., "2-DE proteome analysis of a proliferating and differentiating human neuronal stem cell line (ReNcell VM)," Proteomics, vol. 6, no. 6, pp. 1833-1847, 2006.

[50] K. Kultima, A. M. Nyström, B. Scholz, A. L. Gustafson, L. Dencker, and M. Stigson, "Valproic acid teratogenicity: a toxicogenomics approach," Environmental Health Perspectives, vol. 112, no. 12, pp. 1225-1235, 2004.

[51] F. Ikeda and I. Dikic, "Atypical ubiquitin chains: new molecular signals. "Protein Modifications: Beyond the Usual Suspects” Review Series," EMBO Reports, vol. 9, no. 6, pp. 536$542,2008$.

[52] J. B. Tomas and R. J. Wyman, "Mutations altering synaptic connectivity between identified neurons in Drosophila," Journal of Neuroscience, vol. 4, no. 2, pp. 530-538, 1984.

[53] C. E. Oh, R. McMahon, S. Benzer, and M. A. Tanouye, "bendless, a Drosophila gene affecting neuronal connectivity, encodes a ubiquitin-conjugating enzyme homolog," Journal of Neuroscience, vol. 14, no. 5, pp. 3166-3179, 1994.

[54] M. G. Muralidhar and J. B. Thomas, "The Drosophila bendless gene encodes a neural protein related to ubiquitin-conjugating enzymes," Neuron, vol. 11, no. 2, pp. 253-266, 1993.

[55] M. Watanabe, H. Mizusawa, and H. Takahashi, "Developmental regulation of rat Ubc13 and Uev1B genes in the nervous system," Gene Expression Patterns, vol. 7, no. 5, pp. 614-619, 2007.

[56] Y. Zhu, Y. Sun, X. O. Mao, K. L. Jin, and D. A. Greenberg, "Expression of poly $(\mathrm{C})$-binding proteins is differentially regulated by hypoxia and ischemia in cortical neurons," Neuroscience, vol. 110, no. 2, pp. 191-198, 2002.

[57] M. Kiledjian, X. Wang, and S. A. Liebhaber, "Identification of two $\mathrm{KH}$ domain proteins in the $\alpha$-globin mRNP stability complex," The EMBO Journal, vol. 14, no. 17, pp. 4357-4364, 1995.

[58] J. Nio-Kobayashi, H. Takahashi-Iwanaga, and T. Iwanaga, "Immunohistochemical localization of six galectin subtypes in the mouse digestive tract," Journal of Histochemistry and Cytochemistry, vol. 57, no. 1, pp. 41-50, 2009.

[59] Y. Peng, P. H. Yang, S. S. M. Ng, C. T. Lum, H. F. Kung, and M. C. Lin, "Protection of Xenopus laevis embryos against alcoholinduced delayed gut maturation and growth retardation by peroxiredoxin 5 and catalase," Journal of Molecular Biology, vol. 340, no. 4, pp. 819-827, 2004. 

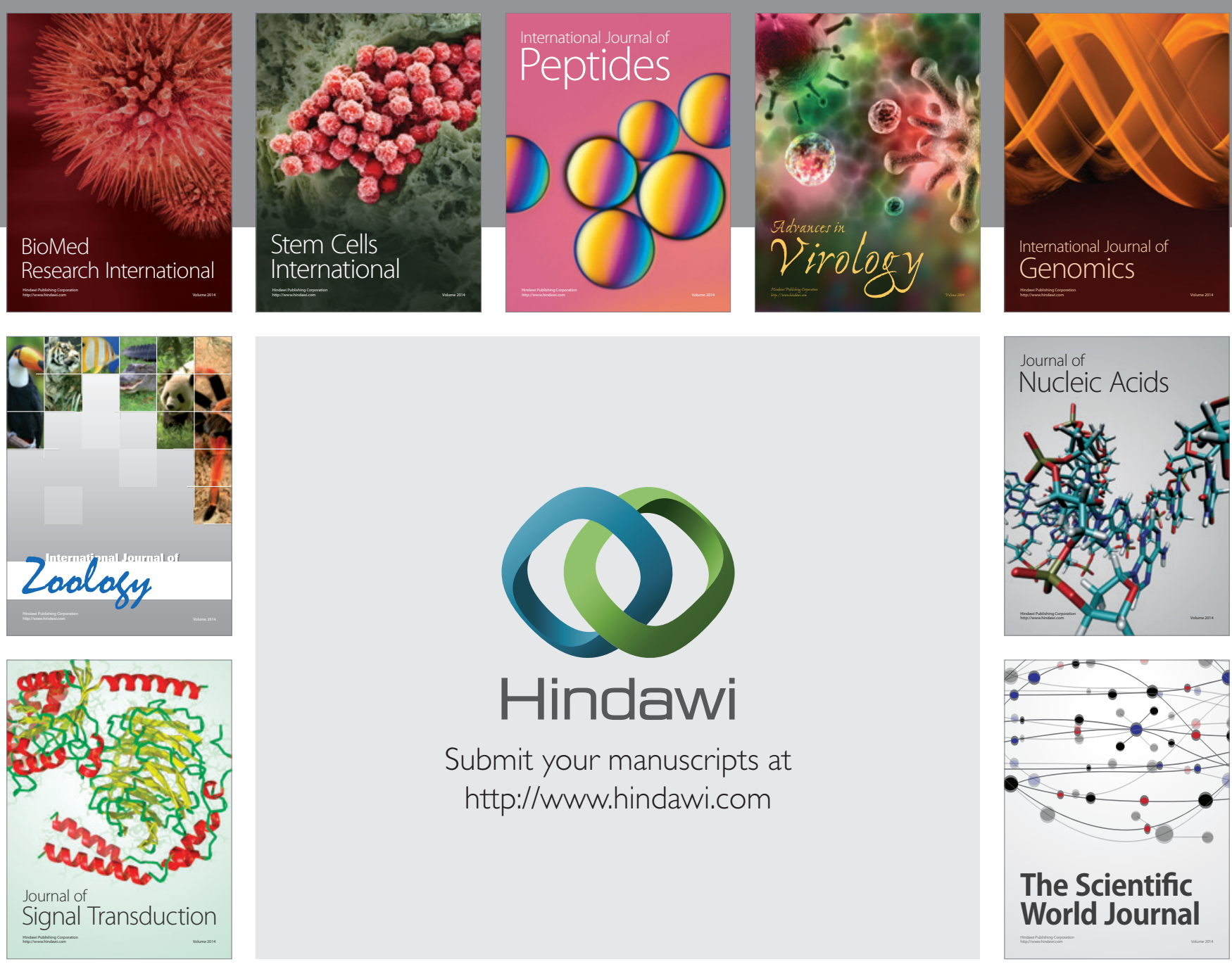

Submit your manuscripts at

http://www.hindawi.com
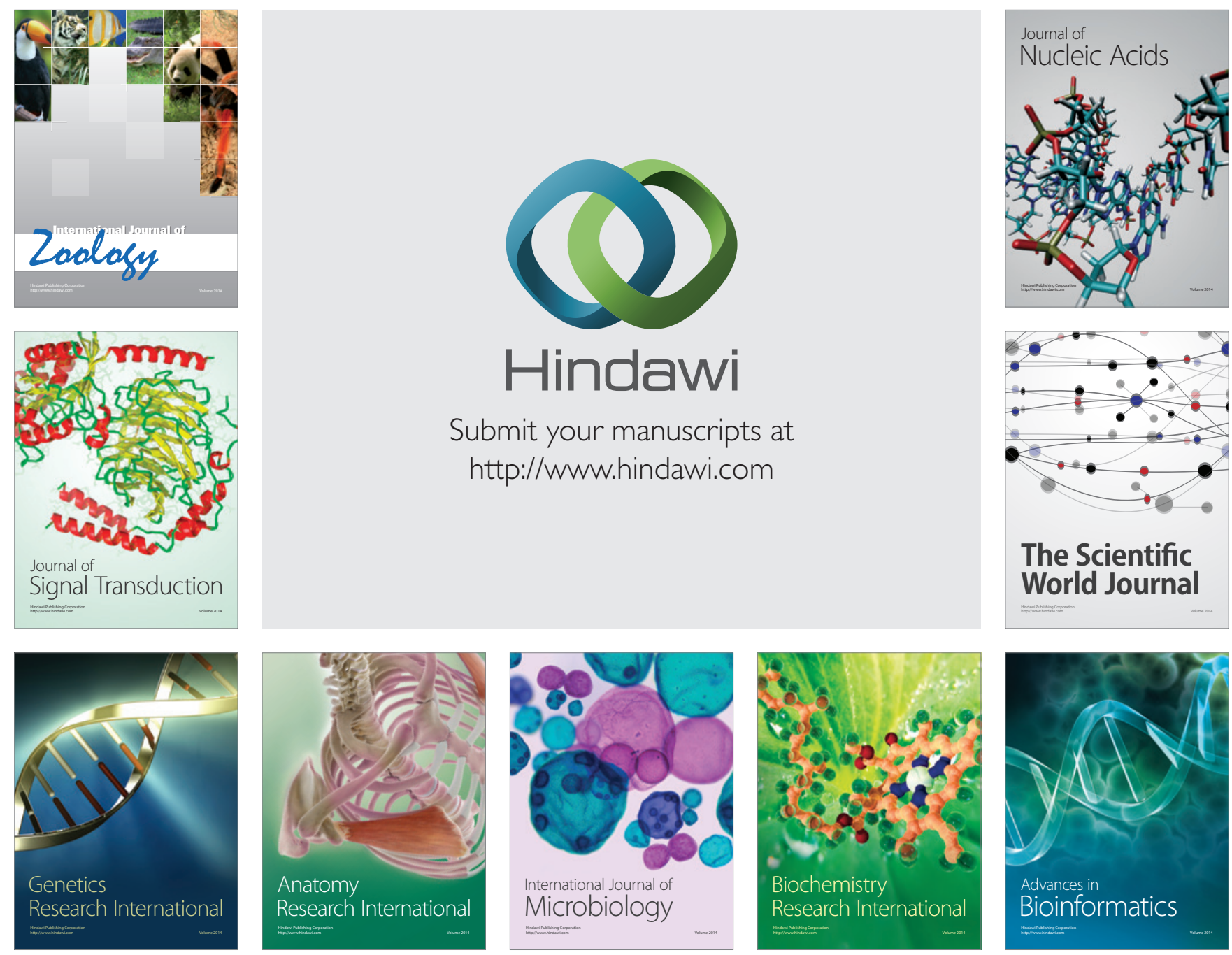

The Scientific World Journal
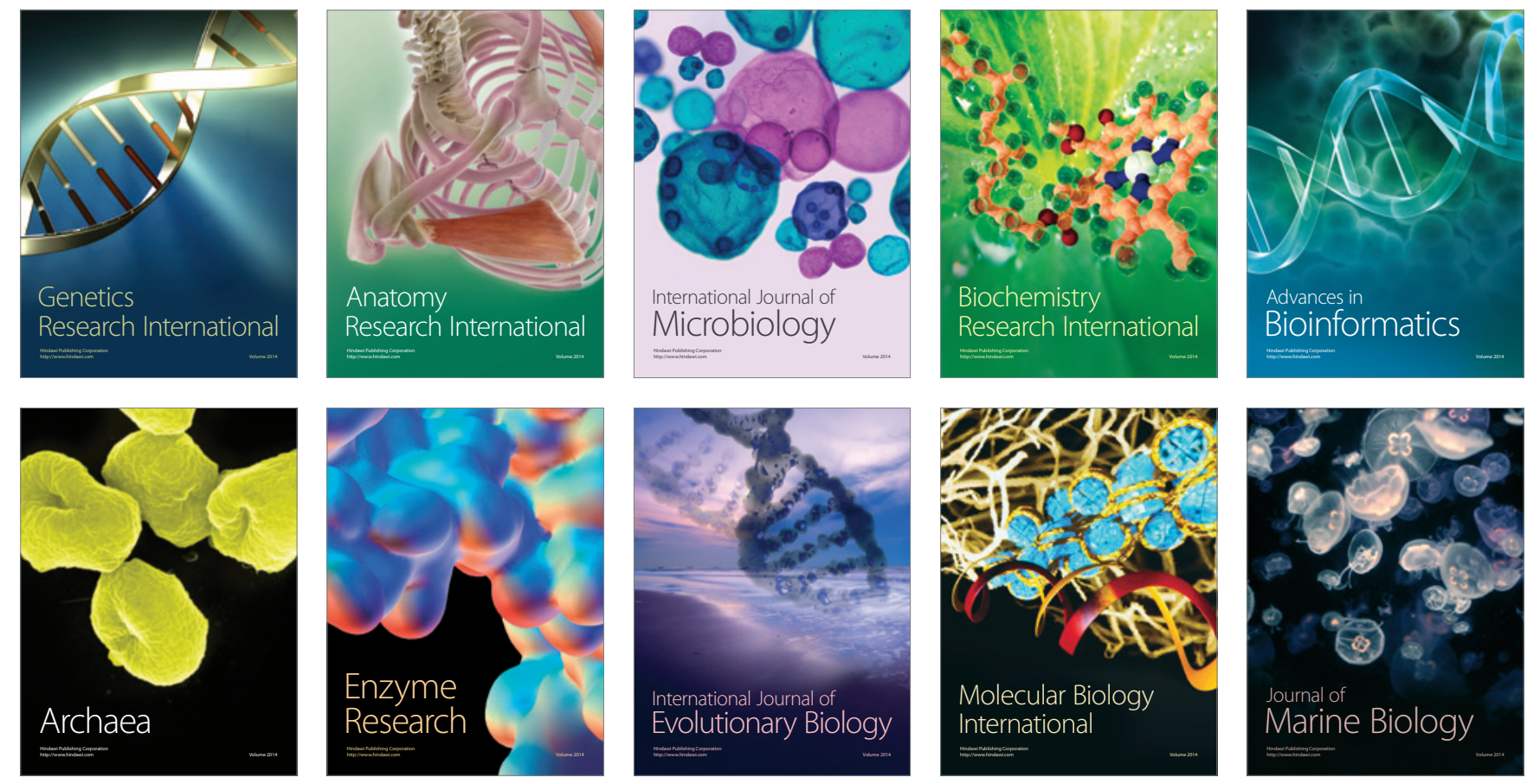